\title{
COMMON FIXED POINT AND INVARIANT APPROXIMATION IN MENGER CONVEX METRIC SPACES
}

\author{
Nawab Hussain, Mujahid Abbas, and Jong Kyu Kim
}

\begin{abstract}
Necessary conditions for the existence of common fixed points for noncommuting mappings satisfying generalized contractive conditions in a Menger convex metric space are obtained. As an application, related results on best approximation are derived. Our results generalize various well known results.
\end{abstract}

\section{Introduction and preliminaries}

Geometric conditions on a mappings and(or) underlying spaces play a crucial role in metric fixed point theory. Although, it has purely metric facet, it is a major branch of a nonlinear functional analysis with close ties to Banach space geometry (see for example, [10]). Several results concerning existence and approximation of fixed point of a mapping rely on convexity hypothesis and geometric properties of Banach spaces. Aronszajn and Panitchapakdi [3] and Menger [19] defined a convexity structure on metric spaces through closed balls and investigated their properties. Khalil [17] further studied existence of fixed points and best approximation in this space.

Recently, Beg and Abbas [4] obtained fixed point and approximate fixed point of nonexpansive and quasi nonexpansive mappings defined on compact convex subsets of uniformly convex metric spaces (see also, [5]). On the other hand, Shahzad [21], O'Regan and Hussain [20] introduced a class of noncommuting mappings called $R$-subweakly commuting mappings, pointwise $R$-subweakly commuting mappings and applied it to $S$-nonexpansive mappings in normed spaces.

This paper deals with the study of common fixed points for $C_{q^{-} \text {-commuting }}$ and uniformly $C_{q}$-commuting mappings in a Menger convex metric space which extends several results in the literature. We also establish results on invariant approximation for these mappings.

Received December 22, 2007.

2000 Mathematics Subject Classification. 47H09, 47H10, 47H19, 54H25.

Key words and phrases. convex metric space, common fixed point, uniformly $C_{q^{-}}$ commuting mapping, asymptotically $S$-nonexpansive mapping, best approximation.

This work was supported by Kyungnam University Research Fund 2007. 
For the sake of convenience, we gather some basic definitions and set out our terminology needed in the sequel.

Definition $1.1([19])$. Let $(X, d)$ be a metric space. It is said to be (Menger) convex metric space if for every $x, y$ in $X, x \neq y, 0 \leq r \leq d(x, y)$,

$$
B[x, r] \cap B[y, d(x, y)-r] \neq \phi,
$$

where $B[x, r]=\{y \in X: d(x, y) \leq r\}$.

Some of the properties of these spaces were introduced by Blumenthal [8]. For further study, we refer to Khalil [16], Berard [7] and references mentioned therein.

Let $X$ be a convex metric space. A nonempty subset $E$ of $X$ is said to be convex, if for all $x, y$ in $E$,

$$
B[x, r] \cap B[y, d(x, y)-r] \subseteq E,
$$

where $0 \leq r \leq d(x, y)$. We note that in general, $B[x, r]$ need not be a convex set in a convex metric space.

Definition 1.2. A convex metric space is said to have property $(A)$, if for every $x, y$ in $X$,

$$
B[x,(1-t) d(x, y)] \cap B[y, t d(x, y)]
$$

is a singleton set for $t \in[0,1]$. We denote this singleton set by $m(x, y, t)$. If $x=y$, then obviously $m(x, y, t)=\{x\}$. The set $B[x, r]$ is convex in a convex metric space with property $(A)$.

A subset $E$ of a convex metric space is said to be $q$-starshaped, if there exists $q$ in $E$ such that for all $x$ in $E$ and $0 \leq r \leq d(x, q)$,

$$
B[x, r] \cap B[y, d(x, q)-r] \subseteq E .
$$

Obviously $q$-starshaped subsets of $X$ contain all convex subsets of $X$ as a proper subclass.

A convex metric space $X$ is said to have property $(B)$ if

$$
d(m(x, y, \lambda), m(x, z, \lambda)) \leq \lambda d(y, z)
$$

and

$$
d(m(y, x, \lambda), m(z, x, \lambda)) \leq(1-\lambda) d(y, z)
$$

for all $x, y, z \in X$ and $\lambda \in(0,1)$. Property $(B)$ is a convex metric space analogue of condition $(I)$ for starshaped metric space of Guay, Singh and Whitfield (see, Definition $3.2[11])$.

Throughout this paper, a metric space $X$ has properties $(A)$ and $(B)$.

Definition 1.3. Let $f, g: X \rightarrow X$. A point $x \in X$ is called;

(1) fixed point of $f$ if $f(x)=x$;

(2) coincidence point of a pair $(f, g)$ if $f x=g x$;

(3) common fixed point of a pair $(f, g)$ if $x=f x=g x$.

$F(f), C(f, g)$ and $F(f, g)$ denote the set of all fixed points of $f$, coincidence points of the pair $(f, g)$ and common fixed points of the pair $(f, g)$, respectively. 
Definition 1.4. Let $E$ be $q$-starshaped subset of a convex metric space $X$ with properties $(A)$ and $(B), q \in F(S)$ and $E$ be both $T$ and $S$ invariant where $T, S: X \rightarrow X$. Put

$$
Y_{q}^{T x}=\left\{y_{\lambda}: y_{\lambda}=m(q, T x, \lambda) \text { and } \lambda \in(0,1]\right\} .
$$

Now, for each $x$ in $X, d\left(S x, Y_{q}^{T x}\right)=\inf _{\lambda \in[0,1]} d\left(S x, y_{\lambda}\right)$. The map $T$ is said to be:

(1) an $S$-contraction, if there exists $k \in(0,1)$ such that

$$
d(T x, T y) \leq k d(S x, S y) .
$$

(2) an asymptotically $S$-nonexpansive, if there exists a sequence $\left\{k_{n}\right\}, k_{n} \geq$ 1 , with $\lim _{n \rightarrow \infty} k_{n}=1$ such that

$$
d\left(T^{n}(x), T^{n}(y)\right) \leq k_{n} d(S x, S y)
$$

for each $x, y$ in $E$ and each $n \in N$. If $k_{n}=1$, for all $n \in N$, then $T$ is known as a $S$-nonexpansive mapping. If $S=I$ (identity map), then $T$ is asymptotically nonexpansive.

(3) R-weakly commuting, if there exists a real number $R>0$ such that

$$
d(T S x, S T x) \leq R d(T x, S x),
$$

for all $x$ in $E$.

(4) $R$-subweakly commuting, if there exists a real number $R>0$ such that

$$
d(T S x, S T x) \leq R d\left(S x, Y_{q}^{T x}\right),
$$

for all $x \in E$.

(5) uniformly $R$-subweakly commuting, if there exists a real number $R>0$ such that

for all $x \in E$.

$$
d\left(T^{n} S x, S T^{n} x\right) \leq R d\left(S x, Y_{q}^{T^{n} x}\right)
$$

(6) $C_{q}$-commuting, if $S T x=T S x$ for all $x \in C_{q}(S, T)$, where $C_{q}(S, T)=$ $U\left\{C\left(S, T_{k}\right): 0 \leq k \leq 1\right\}$ and $T_{k} x=m(q, T x, k)$.

Clearly $\mathrm{C}_{q}$-commuting maps are weakly compatible but the converse is not true (see for example [2]).

(7) A self mapping $T$ on a convex metric space $X$ is said to be affine on $E$, if

$$
T(m(x, y, \lambda))=m(T x, T y, \lambda),
$$

for all $x, y \in E$ and $\lambda \in(0,1)$.

(8) uniformly asymptotically regular on $E$, if for each $\varepsilon>0$ there exists a positive integer $N$ such that $d\left(T^{n} x, T^{n} y\right)<\varepsilon$ for all $n \geq N$ and for all $x$ in $E$.

Definition 1.5. Let $X$ be a metric space and $M$ be a closed subset of $X$. If there exists a $y_{0} \in M$ such that $d\left(x, y_{0}\right)=d(x, M)=\inf _{y \in M} d(x, y)$, then $y_{0}$ is called a best approximation to $x$ out of $M$. We denote by $P_{M}(x)$, the set of all best approximation to $x$ out of $M$. 
Let $M$ be a closed convex subset of a convex metric space with properties $(A)$ and $(B)$ and $x \in X$. Then the set $P_{M}(x)$ is closed and convex [17]. It can also be shown that $P_{M}(x) \subset \partial M$, where $\partial M$ stands for the boundary of $M$.

\section{Common fixed point theorems}

We begin with the definition of uniformly $C_{q}$-commuting mappings.

Definition 2.1 ([12]). Let $E$ be a $q$-starshaped subset of a convex metric space $X$. Let $S, T: E \rightarrow E$ be maps with $q \in F(S)$. Then $S$ and $T$ are said to be uniformly $C_{q}$-commuting on $E$, if $S T^{n} x=T^{n} S x$ for all $x \in C_{q}\left(S, T^{n}\right)$ and $n \in N$.

Clearly, uniformly $C_{q}$-commuting maps on $E$ are $C_{q}$-commuting but the converse is not true.

Example 2.2. Let $X$ be a set of all real numbers with usual metric and $E=[1, \infty)$. Let $T x=2 x-1$ and $S x=x^{2}$, for all $x \in E$. Let $q=1$. Then $E$ is $q$-starshaped with $S q=q$ and $C_{q}(S, T)=\{1\}$ and $C_{q}\left(S, T^{2}\right)=[1,3]$. Note that

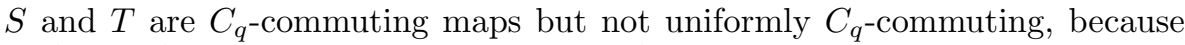
$S T^{2} x \neq T^{2} S x$, for all $x \in(1,3] \subset C_{q}\left(S, T^{2}\right)$.

And also, we know that uniformly $R$-subweakly commuting maps are uniformly $C_{q}$-commuting but the converse does not hold in general, to see this we consider the following example.

Example 2.3. Let $X$ be a set of all real numbers with usual metric and $E=[0, \infty)$. Let $S x=\frac{x}{2}$ if $0 \leq x<1$ and $S x=x$ if $x \geq 1$, and $T x=\frac{1}{2}$ if $0 \leq x<1$ and $T x=x^{2}$ if $x \geq 1$. Then $E$ is 1 -starshaped with $S 1=1$, $C_{q}(S, T)=[1, \infty]$ and $C_{q}\left(S, T^{n}\right) \subseteq[1, \infty]$ for each $n>1$. Note that $S$ and $T$ are uniformly $C_{q}$-commuting but not $R$-weakly commuting for all $R>0$. Thus $S$ and $T$ are neither $R$-subweakly commuting nor uniformly $R$-subweakly commuting mappings.

In this section, existence of common fixed points of $C_{q}$-commuting and uniformly $C_{q}$-commuting, and uniformly $R$-subweakly commuting mappings is established in a Menger convex metric space.

Theorem 2.4. Let $E$ be a nonempty q-starshaped complete subset of a convex metric space, and $T, f$ and $g$ be self mappings on $X$. Suppose $q \in F(f) \cap F(g)$, $T$ is continuous, $f$ and $g$ are continuous and affine on $E, \operatorname{cl}(T(E))$ is compact and $T(E) \subset f(E) \cap g(E)$. If the pairs $\{T, f\}$ and $\{T, g\}$ are $C_{q}$-commuting and satisfy the inequality

$$
\begin{aligned}
d(T x, T y) \leq & \max \left\{d(f x, g y), d\left(f x, Y_{q}^{T x}\right), d\left(g y, Y_{q}^{T y}\right)\right. \\
& \left.\frac{1}{2}\left[d\left(f x, Y_{q}^{T y}\right)+d\left(g y, Y_{q}^{T x}\right)\right]\right\}
\end{aligned}
$$

for all $x, y \in E$, then $T, f$ and $g$ have a common fixed point in $E$. 
Proof. Define $T_{n}: E \rightarrow E$ by

$$
T_{n} x=m\left(q, T x, \lambda_{n}\right),
$$

where $\lambda_{n} \in(0,1)$ with $\lim _{n \rightarrow \infty} \lambda_{n}=1$. Since $E$ is $q$-starshaped, $T_{n}$ is the self mapping on $E$ for each $n \geq 1$. Since $f$ and $T$ are $C_{q}$-commuting and $g$ is affine on $E$ with $f q=q$, for each $x \in C_{n}(f, T) \subseteq C_{q}(f, T)$, we have

$$
f T_{n} x=f\left(m\left(q, T x, \lambda_{n}\right)\right)=m\left(q, f T x, \lambda_{n}\right)=m\left(q, T f x, \lambda_{n}\right)=T_{n} f x .
$$

Thus $f$ and $T_{n}$ are weakly compatible for all $n$. Also since $g$ and $T$ are $C_{q^{-}}$ commuting and $g$ is affine on $E$ with $g q=q, g$ and $T_{n}$ are weakly compatible for all $n$. Also, we have

$$
\begin{aligned}
d\left(T_{n} x, T_{n} y\right) \leq & d\left(m\left(q, T x, \lambda_{n}\right), m\left(q, T y, \lambda_{n}\right)\right) \\
\leq & \lambda_{n} d(T x, T y) \\
\leq & \lambda_{n} \max \left\{d(f x, g y), d\left(f x, Y_{q}^{T x}\right),\right. \\
& \left.d\left(g y, Y_{q}^{T y}\right), \frac{1}{2}\left[d\left(f x, Y_{q}^{T y}\right)+d\left(g y, Y_{q}^{T x}\right)\right]\right\} \\
\leq & \lambda_{n} \max \left\{d(f x, g y), d\left(f x, T_{n} x\right),\right. \\
& \left.d\left(g y, T_{n} y\right), \frac{1}{2}\left[d\left(f x, T_{n} y\right)+d\left(g y, T_{n} x\right)\right]\right\} .
\end{aligned}
$$

By Corollary 3.1 of [9], for each $n \geq 1$, there exists $x_{n}$ in $E$ such that $x_{n}$ is a common fixed point of $f, g$, and $T_{n}$. The compactness of $c l(T(E))$ implies that there exists a subsequence $\left\{T x_{k}\right\}$ of $\left\{T x_{n}\right\}$ such that $T x_{k} \rightarrow y$ as $k \rightarrow \infty$.

From the definition of $T_{k} x_{k}$ and convexity of a metric space, $x_{k} \rightarrow y$. Hence we have $y \in F(T) \cap F(f) \cap F(g)$, by the continuity of $T, f$ and $g$.

Corollary 2.5. Let $E$ be a nonempty $q$-starshaped complete subset of a convex metric space $X$, and $T, f$ and $g$ be self mappings on $X$. Suppose $q \in F(f) \cap$ $F(g), T$ is continuous, $f$ and $g$ are continuous and affine on $E, \operatorname{cl}(T(E))$ is compact and $T(E) \subset f(E) \cap g(E)$. If the pairs $\{T, f\}$ and $\{T, g\}$ are $R$ subweakly commuting mappings satisfying (1), then $T, f$ and $g$ have a common fixed point in $E$.

Corollary 2.6. Let $E$ be a nonempty closed q-star shaped subset of a convex metric space $X, T$ and $S$ be two $R$-subweakly commuting mappings on $E$ such that $T(E) \subset S(E)$, and $c l(T(E))$ is compact where $q \in F(S)$. If $T$ is continuous and $S$-nonexpansive and $S$ is affine on $E$, then $F(T) \cap F(S)$ is nonempty.

Theorem 2.7 ([15], [13]). Let $E$ be a subset of a metric space $(X, d)$ and $S$ and $T$ be weakly compatible self-maps of $E$. Assume that $\operatorname{clT}(E) \subset S(E), \operatorname{clT}(E)$ is complete, and $T$ and $S$ satisfy the inequality

$$
d(T x, T y) \leq h \max \{d(S x, S y), d(S x, T x), d(S y, T y), d(S x, T y), d(S y, T x)\}
$$

for all $x, y \in E$ and $0 \leq h<1$. Then $E \cap F(f) \cap F(T)$ is a singleton. 
Theorem 2.8. Let $E$ be a nonempty closed q-starshaped subset of a convex complete metric space $X$, and $T$ and $S$ be two uniformly $C_{q}$-commuting mappings on $E-\{q\}$ such that $S(E)=E$ and $T(E-\{q\}) \subset S(E-\{q\})$, where $q \in F(S)$. Suppose that $T$ is continuous and asymptotically $S$-nonexpansive with sequence $\left\{k_{n}\right\}$ and $S$ is affine on $E$. And for each $n \geq 1$, define a mapping $T_{n}$ on $E$ by

$$
T_{n} x=m\left(q, T^{n} x, \alpha_{n}\right),
$$

where $\alpha_{n}=\frac{\lambda_{n}}{k_{n}}$ and $\left\{\lambda_{n}\right\}$ is a sequence in $(0,1)$ with $\lim _{n \rightarrow \infty} \lambda_{n}=1$. Then for each $n \in N, F\left(T_{n}\right) \cap F(S)$ is a singleton.

Proof. For all $x, y \in E$, we have

$$
\begin{aligned}
d\left(T_{n}(x), T_{n}(y)\right) & =d\left(m\left(q, T^{n} x, \alpha_{n}\right), m\left(q, T^{n} y, \alpha_{n}\right)\right) \\
& \leq \alpha_{n} d\left(T^{n} x, T^{n} y\right) \\
& \leq \lambda_{n} d(S x, S y) .
\end{aligned}
$$

Moreover, since $T$ and $S$ are uniformly $C_{q}$-commuting and $S$ is affine on $E$ with $S q=q$, for each $x \in C_{n}(S, T) \subseteq C_{q}(S, T)$, we have

$$
S T_{n} x=S\left(m\left(q, T^{n} x, \lambda_{n}\right)\right)=m\left(q, S T^{n} x, \lambda_{n}\right)=m\left(q, T^{n} S x, \lambda_{n}\right)=T_{n} S x .
$$

Thus $S$ and $T_{n}$ are weakly compatible for all $n$. Now, the result follows from Theorem 2.7.

Corollary 2.9. Let $E$ be a nonempty closed $q$-starshaped subset of a convex complete metric space $X$, and $T$ and $S$ be two uniformly $R$-subweakly commuting mappings on $E-\{q\}$ such that $S(E)=E$ and $T(E-\{q\}) \subset$ $S(E-\{q\})$, where $q \in F(S)$. Suppose that $T$ is continuous and asymptotically $S$-nonexpansive with sequence $\left\{k_{n}\right\}$ and $S$ is affine on $E$. And for each $n \geq 1$, define a mapping $T_{n}$ on $E$ by

$$
T_{n} x=m\left(q, T^{n} x, \alpha_{n}\right),
$$

where $\alpha_{n}=\frac{\lambda_{n}}{k_{n}}$ and $\left\{\lambda_{n}\right\}$ is a sequence in $(0,1)$ with $\lim _{n \rightarrow \infty} \lambda_{n}=1$. Then for each $n \in N, F\left(T_{n}\right) \cap F(S)$ is a singleton.

Theorem 2.10. Let $E$ be a nonempty closed $q$-starshaped subset of a convex metric space $X$, and $T$ and $S$ be two self mappings on $E$ such that $S(E)=E$ and $T(E-\{q\}) \subset S(E-\{q\}), q \in F(S)$. Suppose $T$ is continuous, uniformly asymptotically regular and asymptotically $S$-nonexpansive and $S$ is affine on $E$. If $\mathrm{cl}(E-\{q\})$ is compact and $S$ and $T$ are uniformly $C_{q^{-}}$-commuting mappings on $E-\{q\}$, then $F(T) \cap F(S)$ is a singleton.

Proof. From Theorem 2.8, for each $n \in N, F\left(T_{n}\right) \cap F(S)$ is a singleton in $E$. Thus,

$$
S x_{n}=x_{n}=m\left(q, T^{n} x_{n}, \alpha_{n}\right) .
$$


Also,

$$
\begin{aligned}
d\left(x_{n}, T^{n} x_{n}\right) & \left.=d\left(m\left(q, T^{n} x_{n}, \alpha_{n}\right), T^{n} x_{n}\right)\right) \\
& \leq\left(1-\alpha_{n}\right) d\left(q, T^{n} x_{n}\right) \\
& \leq\left(1-\alpha_{n}\right) d\left(q, T^{n} x_{n}\right) .
\end{aligned}
$$

Since $T(E-\{q\})$ is bounded, $d\left(x_{n}, T^{n} x_{n}\right) \rightarrow 0$ as $n \rightarrow \infty$. Now, since $S$ commutes with $T^{n}$ on $C_{q}\left(S, T^{n}\right), x_{n} \in C_{q}\left(S, T^{n}\right)$ and $x_{n}=S x_{n}$, we have,

$$
\begin{aligned}
d\left(x_{n}, T x_{n}\right) & \leq d\left(x_{n}, T^{n} x_{n}\right)+d\left(T^{n} x_{n}, T^{n+1} x_{n}\right)+d\left(T^{n+1} x_{n}, T x_{n}\right) \\
& \leq d\left(x_{n}, T^{n} x_{n}\right)+d\left(T^{n} x_{n}, T^{n+1} x_{n}\right)+k_{1} d\left(S T^{n} x_{n}, S x_{n}\right) \\
& \left.\left.\leq d\left(x_{n}, T^{n} x_{n}\right)+d\left(T^{n} x_{n}, T^{n+1} x_{n}\right)+k_{1} d\left(T^{n} S x_{n}, S x_{n}\right), \alpha_{n}\right)\right) \\
& \left.\leq d\left(x_{n}, T^{n} x_{n}\right)+d\left(T^{n} x_{n}, T^{n+1} x_{n}\right)+k_{1} d\left(T^{n} x_{n}, x_{n}, \alpha_{n}\right)\right),
\end{aligned}
$$

which implies that, $d\left(x_{n}, T x_{n}\right) \rightarrow 0$, as $n \rightarrow \infty$. As $\operatorname{cl}(E-\{q\})$ is compact and $E$ is closed, therefore there exists a subsequence $\left\{x_{n_{i}}\right\}$ of $\left\{x_{n}\right\}$ such that $x_{n_{i}} \rightarrow x_{0} \in E$ as $i \rightarrow \infty$. By the continuity of $T$, we have $T\left(x_{0}\right)=x_{0}$. Since $T(E-\{q\}) \subset S(E-\{q\})$, it follows that $x_{0}=T\left(x_{0}\right)=S y$, for some $y \in E$. Moreover

$$
d\left(T x_{n_{i}}, T y\right) \leq k_{1} d\left(S x_{n_{i}}, S y\right)=k_{1} d\left(x_{n_{i}}, x_{0}\right) .
$$

Taking the limit as $i \rightarrow \infty$, we get $T x_{0}=T y$. Thus, $T x_{0}=S y=T y=x_{0}$.

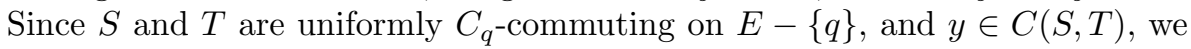
have

$$
d\left(T x_{0}, S x_{0}\right)=d(T S y, S T y)=0 .
$$

This completes the proof.

Corollary 2.11. Let $E$ be a nonempty closed q-starshaped subset of a convex metric space $X$, and $T$ and $S$ be two self mappings on $E$ such that $S(E)=E$ and $T(E-\{q\}) \subset S(E-\{q\}), q \in F(S)$. Suppose $T$ is continuous, uniformly asymptotically regular and asymptotically $S$-nonexpansive and $S$ is affine on $E$. If $\operatorname{cl}(E-\{q\})$ is compact and $S$ and $T$ are uniformly $R$-subweakly commuting mappings on $E-\{q\}$, then $F(T) \cap F(S)$ is a singleton.

Corollary 2.12. Let $E$ be a nonempty closed q-starshaped subset of a convex metric space $X$, and $T$ and $S$ be two self mappings on $E$ such that $S(E)=E$ and $T(E-\{q\}) \subset S(E-\{q\}), q \in F(S)$. Suppose $T$ is continuous, uniformly asymptotically regular and asymptotically $S$-nonexpansive and $S$ is affine on $E$. If $c l(E-\{q\})$ is compact and $S$ and $T$ are $C_{q}$-commuting mappings on $E-\{q\}$, then $F(T) \cap F(S)$ is a singleton.

\section{Invariant approximation results}

Meinardus [18] was the first to employ fixed point theorem to prove the existence of an invariant approximation in Banach spaces. Subsequently, several interesting and valuable results appeared in the literature of approximation 
theory ([1], [21] and [22]). In this section, we obtain results on best approximation as a fixed point of $C_{q}$-commuting and uniformly $C_{q}$-commuting mappings in the setting of a Menger convex metric space.

Theorem 3.1. Let $M$ be a nonempty subset of a convex metric space $X$, and $T, f$ and $g$ be self maps on $X$ such that $u$ is common fixed point of $f, g$ and $T$ and $T(\partial M \cap M) \subset M$. Suppose $f$ and $g$ are affine and continuous on $P_{M}(u)$, where $P_{M}(u)$ is $q$-starshaped with $f\left(P_{M}(u)\right)=P_{M}(u)=g\left(P_{M}(u)\right)$ and $q \in F(f) \cap F(g)$. If the pairs $\{T, f\}$ and $\{T, g\}$ are $C_{q}$-commuting and satisfy for all $x \in P_{M}(u) \cup\{u\}$

$$
d(T x, T y) \leq\left\{\begin{array}{c}
d(f x, g u), i f y=u, \\
\max \left\{d(f x, g y), d\left(f x, Y_{q}^{T x}\right), d\left(g y, Y_{q}^{T y}\right),\right. \\
\left.\frac{1}{2}\left[d\left(f x, Y_{q}^{T y}\right)+d\left(g y, Y_{q}^{T x}\right)\right]\right\}, \text { if } y \in P_{M}(u) .
\end{array}\right.
$$

If $c l\left(P_{M}(u)\right)$ is compact and $P_{M}(u)$ is complete, then $P_{M}(u) \cap F(T) \cap F(f) \cap F(g)$ is nonempty.

Proof. Let $x \in P_{M}(u)$. Then $d(x, u)=d(x, M)$. Note that for any $\lambda \in(0,1)$

$$
\begin{aligned}
d\left(y_{\lambda}, u\right) & =d(m(u, x, \lambda), u) \\
& \leq \lambda d(x, u)<d(x, u)=d(x, M)
\end{aligned}
$$

which shows that, $Y_{u}^{x}=\left\{y_{\lambda}: y_{\lambda}=m(u, x, \lambda)\right\} \cap M$ is empty so $x \in \partial M \cap M$ and $T x \in M$. Since $f x \in P_{M}(u), u$ is common fixed point of $f, g$ and $T$. Therefore, from the contractive condition, we obtain

$$
\begin{aligned}
d(T x, u) & =d(T x, T u) \\
& \leq d(f x, g u)=d(f x, u)=d(u, M) .
\end{aligned}
$$

Thus $P_{M}(u)$ is $T$-invariant. Hence,

$$
T\left(P_{M}(u)\right) \subset P_{M}(u)=f\left(P_{M}(u)\right)=g\left(P_{M}(u)\right) .
$$

The result follows from Theorem 2.4.

Theorem 3.2. Let $M$ be a nonempty subset of a convex metric space $X$, and $T$ and $S$ be two self mappings on $X$ such that and $T(\partial M \cap M) \subset M$, $u \in F(S) \cap F(T)$ for some $u$ in $X$. Suppose $T$ is continuous, uniformly asymptotically regular and asymptotically $S$-nonexpansive and $S$ is affine on $P_{M}(u)$ with $S\left(P_{M}(u)\right)=P_{M}(u), q \in F(S)$ and $P_{M}(u)$ is q-starshaped. If $c l\left(P_{M}(u)\right)$ is compact and $P_{M}(u)$ is complete and $S$ and $T$ are uniformly $C_{q}$-commuting mappings on $P_{M}(u) \cup\{u\}$ satisfying $d(T x, T u) \leq d(S x, S u)$, then $P_{M}(u) \cap$ $F(T) \cap F(S)$ is nonempty.

Proof. Let $x \in P_{M}(u)$. Then $d(x, u)=d(x, M)$. Note that for any $\lambda \in(0,1)$

$$
\begin{aligned}
d\left(y_{\lambda}, u\right) & =d(m(u, x, \lambda), u) \\
& \leq \lambda d(x, u)<d(x, u)=d(x, M)
\end{aligned}
$$


which shows that, $Y_{u}^{x}=\left\{y_{\lambda}: y_{\lambda}=m(u, x, \lambda)\right\} \cap M$ is empty so $x \in \partial M \cap M$ and $T x \in M$. Since $S x \in P_{M}(u), u$ is common fixed point of $S$ and $T$. Therefore, from the contractive condition, we obtain

$$
\begin{aligned}
d(T x, u) & =d(T x, T u) \\
& \leq d(S x, S u)=d(S x, u)=d(u, M) .
\end{aligned}
$$

Thus $P_{M}(u)$ is $T$-invariant. Hence,

$$
T\left(P_{M}(u)\right) \subset P_{M}(u)=S\left(P_{M}(u)\right) .
$$

Now the result follows from Theorem 2.10.

Remark 3.3. (1) Theorem 2.4 extends and improves Theorem 2.2 of AlThagafi [1], Theorem 2.2(i) of Hussain and Jungck [13] and Lemma 2.2 of Shahzad [21].

(2) Theorems 2.8-2.10 extend the results of Beg, Sahu and Dewan [6] to more general classes of mappings.

(3) Following the arguments in [13], we may prove Theorem 2.2-Theorem 2.12 and Theorem 2.14-Corollary 2.16 of Hussain and Jungck [13] for asymptotically $(f, g)$-nonexpansive maps by using Theorem 2.10 .

(4) As an application of Theorem 2.10, we can prove the recent invariant approximation results in $[14,15]$, for uniformly $C_{q}$-commuting, asymptotically $I$-nonexpansive map $T$.

\section{References}

[1] M. A. Al-Thagafi, Common fixed points and best approximation, J. Approx. Theory $8 \mathbf{5}$ (1996), no. 3, 318-323.

[2] M. A. Al-Thagafi and N. Shahzad, Noncommuting selfmaps and invariant approximations, Nonlinear Anal. 64 (2006), no. 12, 2778-2786.

[3] N. Aronszajn and P. Panitchpakdi, Extension of uniformly continuous transformations and hyperconvex metric spaces, Pacific J. Math. 6 (1956), 405-439.

[4] I. Beg and M. Abbas, Fixed points and best approximation in Menger convex metric spaces, Arch. Math. (Brno) 41 (2005), no. 4, 389-397.

[5] _ Inequalities and fixed points in Menger convex metric spaces, Fixed point theory and applications. Vol. 7, 5-15, Nova Sci. Publ., New York, 2007.

[6] I. Beg, D. R. Sahu, and S. D. Diwan, Approximation of fixed points of uniformly Rsubweakly commuting mappings, J. Math. Anal. Appl. 324 (2006), no. 2, 1105-1114.

[7] A. Berard, Characterizations of metric spaces by the use of their midsets: Intervals, Fund. Math. 73 (1971/72), no. 1, 1-7.

[8] L. M. Blumenthal, Distance Geometry, Clarendon Press, Oxford, 1953.

[9] R. Chugh and S. Kumar, Common fixed points for weakly compatible maps, Proc. Indian Acad. Sci. Math. Sci. 111 (2001), no. 2, 241-247.

[10] K. Goebel and W. A. Kirk, Topics in Metric Fixed Point Theory, Cambridge University Press, Cambridge, 1990

[11] M. D. Guay, K. L. Singh, and J. H. M. Whitfield, Fixed point theorems for nonexpansive mappings in convex metric spaces, Nonlinear analysis and applications (St. Johns, Nfld., 1981), pp. 179-189, Lecture Notes in Pure and Appl. Math., 80, Dekker, New York, 1982.

[12] N. Hussain, On strong convergence to common fixed points of uniformly $C_{q}$-commuting maps, submitted. 
[13] N. Hussain and G. Jungck, Common fixed point and invariant approximation results for noncommuting generalized $(f, g)$-nonexpansive maps, J. Math. Anal. Appl. 321 (2006), no. $2,851-861$.

[14] N. Hussain, D. O'Regan, and R. P. Agarwal, Common fixed point and invariant approximation results on non-starshaped domains, Georgian Math. J. 12 (2005), no. 4, 659-669.

[15] N. Hussain and B. E. Rhoades, $C_{q}$-commuting maps and invariant approximations, Fixed Point Theory Appl. 2006, Art. ID 24543, 9 pp.

[16] R. Khalil, Extreme points of the unit ball of Banach spaces, Math. Rep. Toyama Univ. 4 (1981), 41-45.

[17] _ Best approximation in metric spaces, Proc. Amer. Math. Soc. 103 (1988), no. 2, 579-586.

[18] G. Meinardus, Invarianz bei linearen Approximationen, Arch. Rational Mech. Anal. 14 (1963), 301-303.

[19] K. Menger, Untersuchungen über allgemeine Metrik, Math. Ann. 100 (1928), no. 1, $75-163$.

[20] D. O'Regan and N. Hussain, Generalized I-contractions and point wise R-subweakly commuting maps, Acta Math. Sinica., (2007) (in press).

[21] N. Shahzad, Invariant approximations and R-subweakly commuting maps, J. Math. Anal. Appl. 257 (2001), no. 1, 39-45.

[22] S. P. Singh, An application of a fixed-point theorem to approximation theory, J. Approx. Theory 25 (1979), no. 1, 89-90.

NaWAB Hussain

Department of Mathematics

King Abdul AzIZ University

P. O. Box 80203, JedDAh 21589, SAudi Arabia

E-mail address: nhusain@kau.edu.sa

Mujahid ABbas

Department of Mathematics

INDIANA UNIVERSITY

Bloomington, IN 47405-7106

AND

Centre for Advanced Studies in Mathematics and Department of Mathematics LAHORE University of MANAGEMENT SCIENCES

54792-LAHORE, PAKISTAN

E-mail address: mabbas@indiana.edu

JONG KYU KIM

Department of Mathematics, Education

KYUNGNAM UNIVERSITY

Kyungnam 631-701, Korea

E-mail address: jongkyuk@kyungnam.ac.kr 